\title{
KRIPKENSTEIN AND MATHEMATICS AS THE LANGUAGE OF NATURE
}

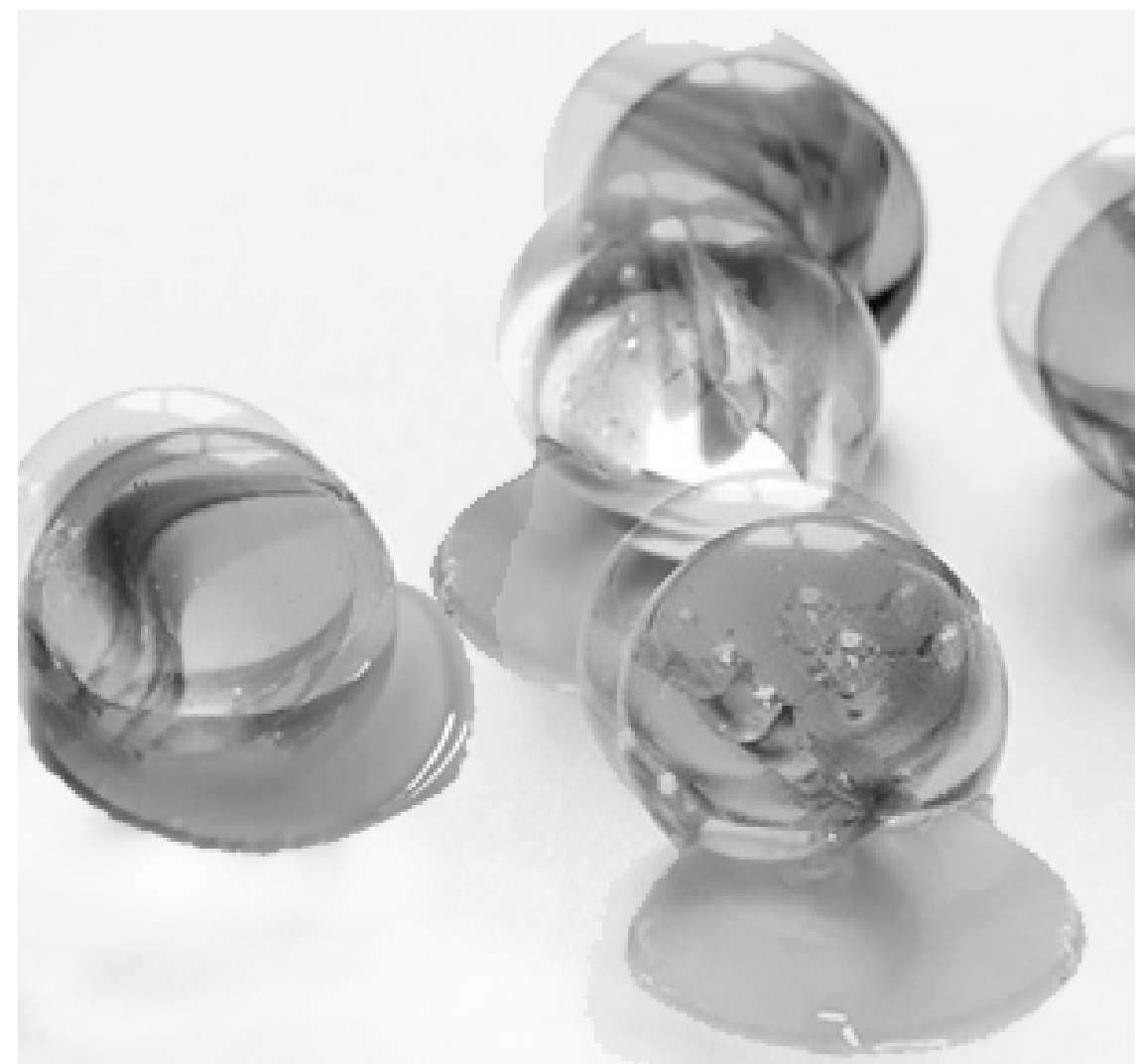

NOUR KHAIRI 


\section{ABSTRACT}

This paper addresses the skeptical paradox highlighted in Saul Kripke's work Wittgenstein on Rules and Private Language. The skeptical paradox stands in the way of many attempts to fix meaning in the rule-following of a language. This paper closely assesses the 'straight solutions' to this problem with regards to another type of language; mathematics. A conclusion is made that if we cannot sufficiently locate where the meaning lies in a mathematical operation; if we cannot describe how it is that we follow a rule in mathematics, we ought to tread lightly in characterising it as the language of nature. 
The logician Saul Kripke undertakes a brave endeavour in exploring Ludwig Wittgenstein's ideas from the Philosophical Investigations (PI). PI takes a closer look at language in the way that many philosophers of language analyze it, as an entity to be understood and dissected in isolation. Wittgenstein argues against the view that language can be understood through ostensive definition (i.e. that words have a direct correspondence to objects in the world). He does this by laying out examples which elucidate the role of language games, the cultural systems that consist of unspoken rules allowing language to work. I propose that language games are conceptually analogous to inside jokes, but on a larger, community scale. Similar to an inside joke, meaning in language is context dependent and fixed by the community of speakers, rather than a corresponding object in the world.

Kripke's book Wittgenstein: On Rules and Private Language is an account of "Wittgenstein's argument as it struck Kripke."1 It is neither an exposition nor a critique of Wittgenstein's ideas, but rather about PI's influence on Kripke. As one would imagine, Kripke finds the implications of PI quite alarming, particularly regarding mathematics. For Wittgenstein to reach his conclusion that meaning in language is fixed within its community, he had to assess rule following in language. A rule allows us to proceed with an utterance; it "guides our hand" when we write or speak. If mathematics is a type of language, it is therefore subject to the same rule following assessment. By locating the rule, we fix the meaning of a word - or a mathematical operation. This assessment leads to the skeptical paradox, presenting us with the challenge of finding the criteria for following a rule correctly. We will see that this activity supposedly threatens the transcendent nature and rigidity of mathematics. Kripke's reaction faces this headfirst by exposing the possible ways we may determine and locate the meaning of a rule in mathematics.

The skeptical paradox position is not exclusive to either Kripke or Wittgenstein. Therefore, a hypothetical skeptic was informally named "Kripkenstein" to represent the famous conundrum of meaning and rule-following. This paper highlights the skeptical paradox along with its possible solutions. Furthermore, it emphasizes the implications of the skeptical paradox on mathematical realism, the position that mathematics is the language of the universe - a highly attractive outlook for formal mathematicians and scientists. I intend to emphasize that we should take mathematical realism with caution, precisely because the skeptical paradox shows us that we cannot assign a straightforward, ostensive meaning to rule-following in mathematics.

1 Saul Kripke, Wittgenstein on Rules and Private Language (Blackwell Publishing, 1982), 5. 
Kripke begins his exploration in a rather Wittgensteinian fashion, by employing a thought experiment aimed at assessing the rule behind an operation like "plus." He asks us to consider an imaginary operation called "quus." Quus, represented by the symbol " $\Theta$," means:

$$
\begin{aligned}
& x \bar{\oplus} y=x+y \text { if } x, y<57 \\
& x \oplus y=5 \text { otherwise }^{2}
\end{aligned}
$$

How do we know that when we add numbers less than 57, we actually mean plus and not quus? If 5 and 6 undergo the function of "+," it is impossible to tell from the result alone whether we used plus or quus. We could think of endless functions like this, where it is impossible to determine whether the specific operation is being followed rather than an alternative one.

This thought experiment seems silly at first, but as Kripke follows Kripkenstein — a skeptic dedicated to questioning where the meaning lies in a rule- he finds that the consequences of this experiment are not so easily brushed aside.

\section{THE PARADOX OF MEANING}

Kripkenstein invokes the rule-following paradox, which Kripke sees as a problem to be solved. Is there some fixed fact that determines whether we are following one rule instead of another? This is the question Kripkenstein demonstrates to be persistent in the face of various counterarguments - commonly known as "straight solutions"- to the skeptical paradox. These counterarguments claim to fix the meaning of a rule (i.e. to give meaning a particular locus, a place where we can point and say "here is the rule, here meaning lies").

One of these claims addresses prior behavior. Meaning is determined by the certainty that in the past, we have performed addition on numerous occasions, and can easily exercise this same skill in the present. In other words, repeatedly solving various examples of addition problems constitutes rule following. However, as alluded to earlier, this falls apart when we introduce a function such as quaddition. When we consider the infinite hypothetical functions we could use that would produce an identical answer, we find there is no external indication of what function was actually being used, or what rule was being followed. Regardless of past or present, Kripkenstein can still pose the question, "How do you know you were not performing quaddition, or skaddition?" This renders the meaning of the operation void. 
Another claim hints at a literal procedure behind the symbol " +." This would be an answer to the seemingly 'fundamental' question 'What constitutes addition?' Kripke presents an example in which you have two heaps of marbles. You count the marbles in the first heap, count the marbles in the second heap, then mix the two heaps together. Finally, after counting the marbles in the resulting heap, you can say with confidence that you have performed addition. ${ }^{3}$ Here, meaning is found in an algorithm. We know the rule was followed correctly if the step-by-step procedure was carried out. Kripkenstein's reply is that this also cannot fix the meaning of the rule. If we look closely at this example, we notice we are simply creating a rule (counting) for following another rule (addition). We are engaging in what Wittgenstein calls "a rule for interpreting a rule." ${ }_{4}$ Counting could have easily been "quounting," and so on. In fact, this attempt is futile since the paradox can be applied ad infinitum for every basic algorithm we characterize as a "break-down" of an operation.

Since we cannot make a distinction between two mathematical rules or functions by interpreting past or present external behavior, a more intuitive approach would be to suggest that meaning is found somewhere in our mental contents. When I say "apple," your mind conjures up an image. Surely, this could tell us something about the meaning of the word "apple." Kripke empathizes with this, admitting that even in writing his comments, he instinctively feels "there is something in [his] mind...the meaning [he] attaches to the "plus' sign." ${ }_{5}$ However, if the goal is to evaluate whether we are using a mathematical function correctly, we need a set standard that one has "understood" the rule. This understanding, as this solution entails, is internal. When we see "+," we feel instructed or "guided" somehow, as though our hand is being forced toward the right answer. However, how can we argue that this "guiding" sensation has roots in our mental contents? The only way to verify this is to find an internal instruction that ensures the correct future usage of plus. How could I possibly check my own correct use of a rule that I set for myself in the past? Kripkenstein taunts us with the infinite ways I could have meant "plus" in the past, that are now unavailable to me in the present. It is not part of my mental experience to deliberately assess every possible function that might have led to my given answer whenever a mathematical problem is presented to me. There is always an ambiguity in claiming to set a specific rule for myself to follow in the future.

Furthermore, this claim renders meaning too subjective. It is impossible for anyone to check my mental contents for a rule that I

3 Kripke, Wittgenstein, 15.

4 Kripke, Wittgenstein, 17.

5 Kripke, Wittgenstein, 22. 
claim is being followed correctly. How then can we all agree on a fixed meaning, when it may very well take its own subjective forms within each individual? We arrive at an epistemological rut, where meaning is an inner state that is impossible to transmit accurately. This defeats the purpose of the challenge posed by Kripkenstein. A fixed meaning cannot be found if we render meaning impossible to share.

The dispositional argument emphasises that we are inclined to give the sum of " $x$ " and " $y$ " when presented with " $x+y$. ." This applies to both past and present instances, and we tend to give the sum of two numbers, rather than their "quum." Here meaning is contained in this disposition itself, in how we are compelled to give one answer over another. ${ }^{6}$ The problem with this is that, along with the finitude of examples I have computed in the past, my dispositions are also finite. Generally, we think that mathematical functions are meant to transcend our human inability to work with extremely large numbers. If presented with such problems, our disposition could easily be to "shrug my shoulders for lack of comprehension" or to give an answer far from the actual sum of the two large numbers. ${ }^{7}$ Dispositions can also vary; my not-so-mathematically-inclined disposition may be to give an answer that is intuitive to me, yet not the "correct" answer. Can meaning comfortably rest on the shaky grounds of our dispositions?

A dispositionalist may try to find a solution for this by proposing that, given the correct tools to compute extremely large numbers, I would be able to give their correct sum. While this is true, Kripkenstein reminds us that this is circular in trying to resolve the skeptical paradox. It implies an error-free, "ideal" scenario in which no possible mistakes are made, and in doing so puts us right back where we started. The subtlety is that the dispositional account conflates description with instruction. It shifts our focus to what we would have answered in the past or future, and despite the accuracy of this prediction, it does not say anything about how we should have answered. The normative element of meaning is then lost when we describe what we would do, rather than what we ought to do. In mathematics, a set correct answer is the crux of assessment. Therefore, we cannot afford to forgo normativity when trying to fix the meaning of a mathematical operation.

One final attempt to defeat the skeptical monster is the appeal that the meaning of a mathematical function such as "plus" exists in the Fregean sense; we simply allude to a shared reference in our use of the symbol "+." This argument suggests a Platonism where we

6 Kripke, Wittgenstein, 24.

7 Kripke, Wittgenstein, 27. 
directly derive fixed meanings of our mathematical symbols from the physical world. We conjure up a symbol that may not mean anything on its own, yet its meaning can be found in what it refers to: a real mathematical object. Here, meaning is considered foundational and irreducible. From a verificationist perspective, it is clear that this is impossible to prove. Strangely enough, this is the main assumption through which the position that mathematics is the language of nature falls out. How would Kripkenstein respond to this? The skeptical paradox by no means denies a shared meaning for a mathematical operation. However, this view suggests that addition corresponds in some "true" way to the function "+." Where is the rule that determines this? This concerns Kripkenstein, and, so far, he has not been fully satisfied by the aforementioned straight solutions.

The skeptical paradox seems to expose various holes in our conception of where the meaning of a rule lies. I urge you to notice that this debate between Kripke and the skeptic has been a process of arguing by elimination. Various solutions have been considered for the skeptical paradox that do not seem to give a satisfactory answer to the question of meaning, yet it is possible that we have overlooked a different solution, or particularities about one solution that go beyond the scope of this paper. However, it is clear that Kripkenstein seems to imply that there is simply no fact that determines the meaning of a function. There is no fact that can distinguish between "plus" and "quus" on the basis of correctness. This is not an epistemological argument that claims we simply cannot know which function is "correct." Rather, he makes an even stronger-almost ontological claim - that no fact exists inside us or out in physical reality that urges us to follow the right function or the right rule. This is quite radical, but still open to the challenge of more solutions that will attempt to "fix meaning."

\section{MATHEMATICS AND THE COMMUNITY}

In true Wittgensteinian fashion, we can direct our focus to the way we actually use mathematical operations. Despite Kripkenstein's paradox, we can easily recall moments when we were corrected for not following a rule correctly in mathematics. In fact, this seems to be an area where it is clearest which answers are "right" or "wrong," at least to expert mathematicians. However, as we saw earlier, the skeptical paradox demonstrates the futility of attempting to fix meaning in a mathematical rule, despite how easily it works in the real world. In PI, Wittgenstein uses daily examples and thought experiments to emphasize that the meaning of words is fixed within the language games of a community of speakers. The same would be true for 
mathematics. It works within the community of mathematicians, and for everyone who has agreed to play the "language-game of mathematics." This is most likely the position that Wittgenstein would take: we find meaning in the usage of the symbol "plus," a symbol that is fixed over time by the members of the community that use it. We can call this the Wittgensteinian solution.

Think of the emergence of imaginary numbers. As the name entails, these are numbers that include an "imaginary" constituent $i$, where,

$$
i=\sqrt{ }-1 .
$$

The constituent $i$ helps us solve problems that would otherwise be deemed impossible. Bombelli (1572) was the first to propose using $i$ to solve problems. From this, imaginary numbers began to gain acceptance in mathematics as they moved away from being a sleight of hand for solving problems, to their own respected mathematical entities. A dedicated mathematical realist may suggest that Bombelli was guided to invent such an entity. However, we can see that the skeptical paradox still permeates by asking how was he guided to do so.

If we seriously consider the Wittgensteinian skeptical solution, we can say that the meaning of $i$ is fixed in the community of mathematicians who believed Bombelli and adopted $i$ as a legitimate way to solve tough quadratics. It is obvious to see how detrimental this is to mathematical realism, since it places the meaning of mathematics and its rigor only in the hands of its community members. Interestingly enough, one of the famous mathematicians in Bombelli's time period was Descartes, who wrote about the imaginary numbers in La Géométrie:

neither the false nor the true roots are always real, sometimes they are only imaginary, that is to say one may imagine as many as I said in each equation, but sometimes there exists no quantity corresponding to that one imagines. ${ }^{8}$

Descartes perfectly presents this problem: numbers like $5 i$ and $7 i$ are inherently imaginary, yet are being treated as though they correspond to particular entities, gaining equal consideration as the "real numbers." Here we see the lack of correspondence to truth values, in fact, the lack of required truth values for mathematics to "work." It is easy to imagine many branches of mathematics arising this way, slowly gaining acceptance from a growing community. We can say that this does it (i.e. this fixes the meaning of a mathematical operation), the implications of which are at direct odds with the notion that mathematics is the language of nature. Consider two communities, "A" follows "plus" the way we do, while "B" follows "quus." If I

8 René Descartes, La Géométrie, appendix to Discours de la méthode, (1637). 
belong to community A, then my answer " 125 " to the question "What is $68+57$ ?" is correct. This means " 5 " is just as correct had I belonged to community $\mathrm{B}$. This societal aspect to the correctness of a mathematical statement shakes the mathematical realism image of deriving meaning in a Platonist sense. Wittgenstein himself notes:

It is interesting to know how many vibrations this note has. But it took arithmetic to teach you this question. It taught you to see this kind of fact. Mathematics, I want to say, teaches you, not just the answer to a question, but a whole language-game with questions and answers. ${ }^{9}$

Wittgenstein implies that when we "do maths," we do not ask questions which have answers that are inspired externally from the natural world, but rather we ask and answer questions within mathematics itself. Mathematics provides us with the lenses through which we recognize mathematical facts. The meaning-fixing domain is comprised of the practice and the members of the community; answers are brought forth by the community and integrated into the practice. So far, it seems as though we can fix meaning after all. We can fix it in the community of speakers over time. Can we say then that this solves the skeptical paradox and be done with it? Many have argued that this solution is still superficial with regards to the skeptical paradox and fails to capture its full implications. For instance, Michael Morris emphasizes that the community-based solution does not point to truth conditions, but only assertibility conditions..$^{10}$ Belonging to community A gives me the right to assert that 125 is the correct answer, but it is still not certain that this is the right answer. We move away from a realist fact, to an assertion based on agreement. For Morris, this makes the Wittgensteinian solution insufficient to fully tackle Kripkenstein's paradox.

Even further, Morris suggests that Kripkenstein may be implying that there is no determinate way of defining the borders of a community. That is, belonging to one community or another is arbitrary. ${ }^{11}$ In our "quus" example, if my question involved numbers less than 57, there is no indication that I belonged to community A or $\mathrm{B}$ based on my answer, and therefore no way to check my assertion.

I propose that the skeptical paradox is not fully resolved yet. Meaning in the factual sense cannot be fixed in our prior behaviour, mental contents, dispositions, a Platonist "outer" world, or more radically, the community we belong to. Kripkenstein continues to

9 Ludwig Wittgenstein and G.H. Von Wright, Remarks on the Foundations of Mathematics (Oxford: Basil Blackwell, 1956), 15.

10 Michael Morris, An Introduction to the Philosophy of Language (New York: Cambridge University Press, 2007), 271-91.

11 Morris, Philosophy of Language. 
nudge us in the direction that meaning cannot ever be fixed. If we take a practical, Wittgensteinian approach to this issue, we can shrug our shoulders and say, "So what?" Meaning is still determined and has been defined very clearly over time by the community of speakers, allowing us to judge a mathematical answer and agree to it cross-culturally with incredible accuracy. We need not worry about the truth value of a mathematical function, since it is irrelevant to the very activity we are engaged in: to answer a question in mathematics through mathematical language games. It becomes extremely challenging, however, when we want to say that mathematics is a factual description of the natural world, since we cannot point to where the meaning lies in following a mathematical rule. If I point to the community of mathematicians, this still gives us no realist fact about meaning and only a relative evaluation of correctness. Therefore, we cannot say with any factual justification that the language of mathematics extends beyond our particular community of mathematicians, let alone that it extends to the universe.

Whether this is alarming or not is a matter of perspective. If you are a true Wittgensteinian, fixing the meaning of a rule is unnecessary because the rule works naturally and consistently within our community regardless - granted we adopt its language games. We must therefore simply accept our inability to fix meaning by analysing both linguistic and mathematical rules in isolation. According to the skeptical paradox, such an activity fails to illuminate anything about meaning and rule-following. The paradox urges us toward the conclusion that meaning cannot be fixed in a rule, which could be worrisome if you are a realist about mathematics. The task, therefore, would be to propose an entirely new solution to the paradox. However, if you can abandon the notion of mathematical realism, the "sociological," Wittgensteinian view would be that analysis of rules is simply unnecessary. The rigor of mathematics is still maintained, granted we are ready to accept its societal origin. I can say, undoubtedly, that the skeptical paradox reminds us to be more careful before claiming that any human-made system of symbols should correspond to, or be the language of, nature herself. 


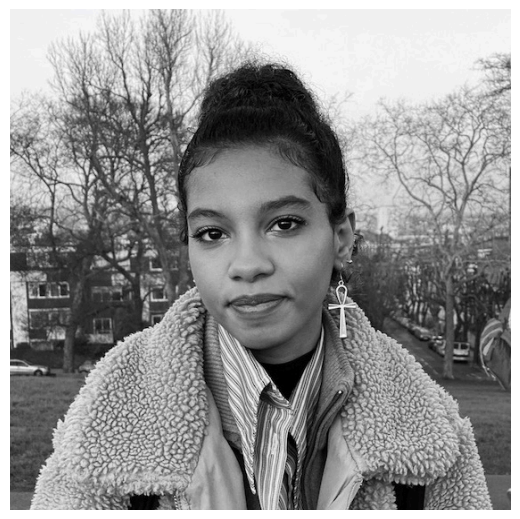

Nour Khairi is a third-year student at University College Utrecht in the Netherlands, majoring in Physics and minoring in Philosophy. They love exploring where philosophy intersects with physics and mathematics. Originally Sudanese, born and raised in Qatar, Nour is a passionate writer and traveller. 
